\title{
Is Fe-Ni alloy in Ordinary Chondrites formed by precursors partial melting?
}

\author{
GUILLAUME FLORIN ${ }^{1}$, OLIVIER ALARD ${ }^{1}$, BEATRICE
} LUAIS $^{2}$ AND TRACY RUSHMER ${ }^{1}$

${ }^{1}$ Macquarie University

${ }^{2}$ Université de Lorraine, CNRS, CRPG

Presenting Author: gf.florin@gmail.com

Ordinary chondrites (OCs), the most common meteorites on Earth, are undifferentiated objects composed of silicate, metal, and sulphide. Despite their widespread occurrence, processes that lead to their formation remain largely enigmatic. Therefore, understanding OCs component formation is one of the key features to bring about new constraints on early protoplanetary disk evolution. Within these components, metal formation is one of the most controversial subject. Numerous hypothesis have been formulated: (1) formation by $\mathrm{FeO}$ reduction from silicate precursors [1]; (2) complex condensation mixing processes [2]; and (3) troilite desulfurization [3]. Recent LA-ICP-MS in-situ measurements [4] tend to demonstrate that variability of metal composition in $\mathrm{L} / \mathrm{LL}$ groups results from melting-crystallisation of metal precursors. Currently, no consensus exists, therefore we have carried out a detailed in-situ investigation of siderophile elements (PGEs, W, Mo, Au, Cu, Ge, Ga) within the metal phase in $\mathrm{H}$ chondrites.

We have chosen four $\mathrm{H}$ unequilibrated and five $\mathrm{H}$ equilibrated OCs to explore metal pre-accretionary evolution and infer the effect of metamorphism. We have conducted analyzes using a LA-ICP-MS, G2 photon machine $193 \mathrm{~nm}$ HelEx-Cell with a 7500 Agilent ICP-MS at Macquarie University.

We highlight siderophile elemental variations between metal grains in H3. Here, metamorphism homogenised the variations, demonstrating the important link with pre-accretionary processes. Negative correlations between Ge/Ir and PGEs, allow the classification of metal grains in $\mathrm{H} 3$ into three groups: high Ge/Ir-low HSEs, low Ge/Ir-high HSEs, and intermediate compositions. Concurring with previous studies on L/LL [5], we show that compositional range in H OCs can be explained by melting of a single metallic precursor containing up to $1.5 \mathrm{wt} . \%$ $\mathrm{C}$ and 0-12 wt.\% S. Yet, metal melting cannot explain W, Mo, Ga compositions, which better reflect various redox states at grain scale during heating events. We suggest that metal in $\mathrm{H}$ OCs formed following partial melting of metal-silicate precursors under various oxidation states, buffered by the evaporation of oxidizing component(s) which is/are heterogeneously distributed.

[1] Kong and Ebihara (1997), GCA 61(11), 2317-2329. [2] Horan et al. (2009), GCA 73(22), 6984-6997. [3] Hewins et al. (1997), Antarctic Meteorite Research 10, 275-298. [4] Okayabashi et al. (2019), GCA 244, 197-215. 